\title{
What kind of journal should Episodes be?
}

Episodes was the brainchild of former IUGS Secretary General (later President) W. W. Hutchison of Canada, who saw clearly the need for the Union to communicate effectively and regularly with its commissions and affiliated organizations and with members of the international community of earth scientists. The journal had two progenitors. After IUGS was founded in 1961, its initial outreach medium was the Circular Letter. This was replaced later in the decade by the Geological Newsletter. which in turn was supplanted by Episodes in 1978. The present journal will, therefore, shortly come of age. During its formative years, it has found an editorial home in four countries, successively with the national geological surveys of Canada, the United States, and the United Kingdom, and now with the Ministry of Geology and Mineral Resources of China.

When it was launched, Episodes was an immediate success. Its number of subscribers rose steadily; it dramatically heightened the profile of IUGS in the global earth-science community; it made the Union a reality in many small countries of the developing world, which began to receive the journal through a special agreement with UNESCO; and it was acclaimed in the International Council of Scientific Unions, to which IUGS belongs. But despite the commitment and support of many parties around the world - not least the organizations that have been hosts to Episodes' editorial office - the economic realities of recent years have led to variations in the quality, scope, publication schedule, and subscription base of the journal to the point that it recently has come under increasing scrutiny by the Advisory Board on Publications (ABP) and the Executive Committee of IUGS. Central to the discussions have been two interdependent questions: how may Episodes better serve the interests of IUGS and the earth-science community and, to do that, what kind of journal should Episodes be?

There has been no shortage of suggestions but a distinct lack of concensus. Some committee members have argued that raising the scientific quality is the key. They advocate publishing more externally reviewed, high-quality, original articles on the 'leading edge' of modern, international earth science, especially earth-system science, even going so far as to suggest that Episodes seek ultimately to supersede Nature and Science in this respect. In sharp contradistinction, an attendee at the IUGS Council Meeting during the 30th International Geological Congress last year recommended that Episodes should become, 'a newsletter, more than...another scientific journal.' Another attendee commented that it should not try to serve experts in the earth sciences: it should communicate widely "more general information' and 'should not try to compete with Nature.' Within the IUGS' committees, others stress the importance of Episodes to geological organizations of developing countries, for some of which it is their sole link to the outside world. They appeal, therefore, for a mixed content, including assessments of new developments in the earth sciences, news about IUGS, reviews of new books and maps, conference reports, and a calendar of forthcoming international meetings. Still others again, deeply concerned about issues of 'geoscience and society,' believe that Episodes should devote the bulk of its content to environmental earth science and related topics - energy, mining practices, water supply, waste disposal, crop-generating soil production, natural disasters, and such like. Not all of these suggestions are completely contradictory, of course. Some committee members opine that, by publishing a series of succinct well-written reviews to expose where the 'leading edge' of research in the earth sciences now lies, a valuable service would be provided to teachers and practitioners in developing countries. Others point out that a much more valuable service would be rendered these same parties if the articles and reviews in Episodes were to deal with the kinds of issues that must have pride of place in the developing countries, viz, those of 'geoscience and society.' Still others stress the obvious ties between these issues and emphasis on the earth-system approach - a point forcefully made by W. S. Fyfe in the lead article of the first issue for 1997.

There seems to be unanimity on only two points, both of which are related to, but a step removed from, policy on content. The first is that Episodes must be restored to a regular quarterly publication of about 80 pp. per issue and be distributed on time. Editor Zhang Hongren, Managing Editor Hou Hongfei, and the other members of their five-person production team already have taken steps to meet these objectives. The Ministry of Geology and Mineral Resources has generously provided for the Editorial Office with fast communication aids and printing outlets to achieve this end. The second point is that Episodes and its Editor should have the support of an internationally based Editorial Board, the members of which not only monitor the scientific quality of the journal's contents but actively solicit the best of available contributions from all parts of the world. This Editorial Board is in process of being appointed.

On the question of the journal's content and editorial thrust, the ABP has recommended that, for the time being, the answer lies in compromise. The Board has not done so negatively, because compromise avoids decision-making and offers the path of least-concerted resistance, but positively, because only by compromise can IUGS, through Episodes, serve the widest cross-section of its international constituency for at least the next few years. By identifying distinct sections within the journal, there can be a place for original articles and reviews on important developments in the earth sciences, provided these have international appeal or substance; a place for scientific notes on new discoveries and their potential import, prior to their full substantiation in the archival literature; a place for contributions on the all-important issues of 'geoscience and society'; a place for describing new conclusions reached by the IUGS' commissions, subcommissions, and working groups and for reporting other IUGS news; and a place for relevant book and map reviews, conference reports, letters to the editor, and a calendar of forthcoming events. This does not mean that the ABP wants Episodes to remain in statuo quo $\longrightarrow$ far from it. For example, the overall quality of articles and reviews should be improved: a concerted effort is needed to attract more scientific notes on new discoveries; many more contributions are warranted on the critical role the earth sciences must play in sustaining the geosphere in an overpopulated world thirsty for natural resources; and there is a need for more regular and substantial sections in which new books and maps and recent conferences, relevant to international earth science, are critically and informatively reviewed.

IUGS wants its journal to serve you, the readership, and would like to have your views on 'What kind of journal should Episodes be?' Do you agree with the ABP's compromise policy or not? Are you aware of other important considerations regarding Episodes that IUGS seems not to have considered? Further information on the journal (with names, addresses, and communication numbers of IUGS officials) is available inside the front cover of this issue or in the new, constantly updated, user-friendly IUGS entry in the worldwide web (http://www.iugs.org). Please communicate your views to any one of Robin Brett, IUGS President; Zhang Hongren, Episodes' Editor; John Aaron, IUGS Webmaster; or me. Of equal or greater value would be a demonstration by example of what you would like to see published: prepare an appropriate contribution for the journal and send it to the Editor for his consideration.

W. G. E. Caldwell Chairman, $A B P$ 